\title{
Article \\ Sustainable Combination Mechanism for Catalysts: A Game-Theoretical Approach
}

\author{
Kelvin H.-C. Chen ${ }^{1}\left(\mathbb{D}\right.$, Jong-Chin Huang ${ }^{1, * \mathbb{C}}$ and Yu-Hsien Liao ${ }^{2}$ \\ 1 Department of Applied Chemistry, National Pingtung University, Pingtung 900, Taiwan; \\ kelvin@mail.nptu.edu.tw \\ 2 Department of Applied Mathematics, National Pingtung University, Pingtung 900, Taiwan; \\ twincos@ms25.hinet.net \\ * Correspondence: hjc@mail.nptu.edu.tw; Tel.: +886-8-766-3800
}

Citation: Chen, K.H.-C.; Huang,

J.-C.; Liao, Y.-H. Sustainable

Combination Mechanism for Catalysts: A Game-Theoretical Approach. Catalysts 2021, 11, 345 https://doi.org/10.3390/ catal11030345

Academic Editor: Carlo Santoro

Received: 8 February 2021

Accepted: 3 March 2021

Published: 8 March 2021

Publisher's Note: MDPI stays neutral with regard to jurisdictional claims in published maps and institutional affiliations.

Copyright: (C) 2021 by the authors. Licensee MDPI, Basel, Switzerland. This article is an open access article distributed under the terms and conditions of the Creative Commons Attribution (CC BY) license (https:/ / creativecommons.org/licenses/by/ $4.0 /)$.

\begin{abstract}
Catalysts, artificially or naturally generated, are often considered to be important factors in numerous chemical processes. Although each catalyst can act under its own characteristics, the efficiency of chemical interactions can be enhanced by a balanced combination of different catalysts. On the other hand, many game-theoretical results have been widely applied to seek the optimal or balanced state for efficiency regulation, resource control, portfolio allocation, and behavior simulation in modern academic literature. Based on game-theoretical approaches under actual chemical and biochemical environments, this article aims to analyze, construct, simulate, and derive the most efficient optimal or balanced combinations for a group of catalysts with different conditions and actions. In this article, a power index is proposed by simultaneously focusing on the factors and its active levels. In order to analyze the accuracy and rationality of this power index, we adopt usual axioms to offer some characterizations. In conjunction with the constructed game-theoretical results that are related to chemical and biochemical environments, this article further analyzes, verifies, and demonstrates the validity, accuracy, feasibility, plausibility, and applicability of the combination of catalysts with different conditions and actions.
\end{abstract}

Keywords: factor; active level; power index; combination for catalysts

\section{Introduction}

Catalysts, artificially or naturally generated, are often considered to be important factors in numerous chemical processes. There are many different types of catalysts, including enzymatic, photocatalytic, and chemical catalysts. Although each catalyst can act under its own characteristics, the efficiency of chemical interactions can be enhanced by a balanced combination of different catalysts. In addition to improving reversible effects, the damage of irreversible effects can also be mitigated. To assess the efficacy of the combination of components, empirical prediction, the imitation of similar procedures, or inference of posterior results, as well as the construction, simulation, and derivation of academic theories in different fields, may be conducted to verify the accuracy, feasibility, and plausibility of such a combination. Related investigations have been wildly introduced. For example, mixed oxides were synthesized by the co-precipitation of a $\mathrm{Cu}$ source in combination with $\mathrm{Al}, \mathrm{Fe}$, or $\mathrm{Mn}$ corresponding salts as precursors. In order to crystallize the phases and to mimic the reaction conditions of the catalytic application, Everbroeck et al. [1] considered reactive results when the materials were calcined at $600{ }^{\circ} \mathrm{C}$ and $100{ }^{\circ} \mathrm{C}$ respectively; and, Gebresillase et al. [2] investigated the influence of metal loading, metal combination, and ratio on the activity and selectivity of the catalysts. Ni-based catalysts were also found to be highly active for the related reaction; the review article, due to Losada-Garcia et al. [3] provided an overview of the novel and recent achievements in enzyme cascade processes catalyzed by multi-enzymatic or chemoenzymatic systems. The selected examples that were collected the advances related to the application of the sequential use of enzymes in 
natural or genetically modified combination; the study, due to Malgas and Pletschke [4] showed that using the activity of endo-mannanases displayed with model substrates is a poor predictor of their activity and synergism on complex lignocelluloses. Applying the endo-mannanases in combination with $\mathrm{CTec} 2$ for lignocellulose degradation led to synergism, with a 1.3-fold increase in reducing sugar release as compared to when CTec2 was used alone; advances in the Aspergillus-specific molecular toolkit and combination of several engineering strategies resulted in strains that were able to generate high titers of recombinant fungal proteins. Ntana et al. [5] described key steps in protein synthesis and secretion that may limit the production efficiency in Aspergillus systems and present genetic engineering approaches and bioprocessing strategies that have been adopted in order to improve recombinant protein titers and expand the potential of Aspergilli as competitive production platforms; single-atom catalysts (SACs), consisting of metals atomically dispersed on a support, are considered as advanced materials bridging homogeneous and heterogeneous catalysis, representing the catalysis at the limit. The enhanced performance of these catalysts is due to the combination of distinct factors. The study of Pérez-Mayoral et al. [6] summarized the most recent advances in terms of both synthetic strategies of producing porous carbon-derived SACs but also its application to green synthesis of highly valuable compounds, an area in which the homogeneous catalysts are classically used.

In modern academic literature, game theory has been widely applied to seek the optimal or balanced state for efficiency regulation, resource control, portfolio allocation, and behavior simulation. Under traditional transferable-usability (TU) circumstances, a usability function is defined by considering all of the subsets among the set of factors. This means that the options available for every factor are either to react fully in a reaction process or not to react at all. However, in real life, regulation, control, allocation, and simulation usually vary relative to each other in response to the rapidly changing interactions between individuals, groups, and environments. Therefore, a multi-choice TU circumstance could be regarded as a natural extension of a traditional TU circumstance in which each factor presents various active levels. On the other hand, the power indexes have been introduced to verify the ability of each factor of a system. Each factor will have a certain amount of active levels, and so its ability will be different. Several power indexes have been proposed under multi-choice TU circumstances. By computing the overall effects for a given factor under multi-choice TU circumstances, Hwang and Liao [7], Liao [8,9] and Nouweland et al. [10] considered different mechanisms, indexes, and related results by respectively applying the notions of the core, the EANSC, and the Shapley value. In this article, we consider a generalization of the pseudo equal allocation of non-separable costs (PEANSC). Under traditional TU circumstances, Hsieh and Liao [11] first adopted the individual index to propose the PEANSC, and further defined a notion of reduced circumstance and its consonance to demonstrate that the PEANSC is a stable power index contenting several useful axioms.

These factors mentioned above raise one motivation:

- whether the pre-existing results of the PEANSC could be extended to analyze, construct, simulate, and derive the most efficient optimal or balanced combinations for a group of catalysts with different conditions and actions under multi-choice behavior. The article is devoted to investigating the motivation. Several main results are, as follows.

- Inspired by the works of Hwang and Liao [12], a generalization of the PEANSC is proposed by simultaneously focusing on the factors and its active levels in Section 2, which we name the multi-choice level-individual index (MLII).

- In order to analyze the accuracy and the rationality of the MLII, we introduce alternative extended properties of Hsieh and Liao [11] and the related consonance to characterize the MLII in Section 3.

- In conjunction with the constructed game-theoretical results that are related to chemical and biochemical environments, this article further analyzes, verifies, and demonstrates the validity, accuracy, feasibility, plausibility, and applicability of the combi- 
nation of catalysts with different conditions and actions in Section 4. Some more applications, interpretations, and comparisons are also offered throughout this article.

\section{Preliminaries}

\subsection{Definitions and Notations}

Let $U F$ be the universe of factors, for example, the set that formed by catalysts throughout the Earth. Any $p \in U F$ is said to be a factor of $U F$, for example, a catalyst of the Earth. For $p \in U F$ and $b_{p} \in \mathbb{N}, B_{p}=\left\{0,1, \cdots, b_{p}\right\}$ could be treated as the active level space of factor $p$ and $B_{p}^{+}=B_{p} \backslash\{0\}$, where 0 denotes no reaction. Assume that $F \subseteq U F$ is the largest set of all factors of an interactive system in $U F$, for example, all the catalysts of a aquatic environment on Earth. Let $B^{F}=\prod_{p \in F} B_{p}$ be the product set of the active level (action) spaces of all factors of $F$. For all $K \subseteq F$, we define $\kappa^{K} \in B^{F}$ is the vector with $\kappa_{p}^{K}=1$ if $p \in K$, and $\kappa_{p}^{K}=0$ if $p \in F \backslash K$. Denote $0_{F}$ as the zero vector in $\mathbb{R}^{F}$.

A multi-choice TU circumstance is a triple $(F, b, C)$, where $F \neq \varnothing$ is a finite set of factors, $b=\left(b_{p}\right)_{p \in F}$ is the vector that presents the highest amount of all active levels for each factor, and $C: B^{F} \rightarrow \mathbb{R}$ is a usability map with $C\left(0_{F}\right)=0$ that assigns, to each $\lambda=\left(\lambda_{p}\right)_{p \in F} \in B^{F}$, the usability that the factors can occur when each factor $p$ reacts at active level $\lambda_{p}$. As $b \in \mathbb{R}$ is fixed throughout this article, we write $(F, C)$, rather than $(F, b, C)$.

Denote the class of all multi-choice TU circumstances by $\Psi$. Given $(F, C) \in \Psi$ and $\lambda \in B^{F}$, we write $A(\lambda)=\left\{p \in F \mid \lambda_{p} \neq 0\right\}, \lambda_{K}$ to be the restriction of $\lambda$ at $K$ for each $K \subseteq F$ and $\|\lambda\|=\sum_{p \in F} \lambda_{p}$.

Given $(F, C) \in \Psi$, let $L^{F}=\left\{\left(p, k_{p}\right) \mid p \in F, k_{p} \in B_{p}^{+}\right\}$. A power index on $\Psi$ is a map $\tau$ assigning, to each $(F, C) \in \Psi$, an element

$$
\tau(F, C)=\left(\tau_{p, k_{p}}(F, C)\right)_{\left(p, k_{p}\right) \in L^{F}} \in \mathbb{R}^{L^{F}} .
$$

Here, $\tau_{p, k_{p}}(F, C)$ is the effect or the power of the factor $p$ when it reacts in a coalition with level $k_{p}$ in $(F, C)$. For convenience, let us consider $(F, C) \in \Psi$ and a power index $\tau$ on $\Psi$, we define $\tau_{p, 0}(F, C)=0$ for each $p \in F$.

Subsequently, we offer a generalized analogue of the pseudo equal allocation of non-separable costs on multi-choice circumstances, as follows.

Definition 1. The multi-choice level-individual index (MLII) of multi-choice circumstances, $\bar{\gamma}$, is the map on $\Psi$, which associates to each $(F, C) \in \Psi$, each factor $p \in F$ and each $k_{p} \in B_{p}^{+}$ the effect

$$
\bar{\gamma}_{p, k_{p}}(F, C)=\gamma_{p, k_{p}}(F, C)+\frac{1}{\|b\|} \cdot\left[C(b)-\sum_{t \in F} \sum_{q=1}^{b_{t}} \gamma_{t, q}(F, C)\right],
$$

where $\gamma_{p, k_{p}}(F, C)=C\left(k_{p}, 0_{F \backslash\{p\}}\right)-C\left(k_{p}-1,0_{F \backslash\{p\}}\right)$ is the individual-level distinction of the factor $p$ from its level $k_{p}-1$ to $k_{p}$. Under the power index $\bar{\gamma}$, factors firstly take its individuallevel distinctions under corresponding levels, and further distribute equally the remaining usability among all the active levels.

\subsection{Motivating and Practical Examples}

In order to illustrate how the framework of multi-choice TU circumstance and the MLII could be applied and to ensure that its meaning is more clear and visible, we offer several motivating and practical examples on reaction processes of combinations for catalysts, as follows.

Example 1. Let $(F, C) \in \Psi$ and $F$ be a collection of factors of reaction processes of combinations for catalysts. Assume that the amount of the active levels (reaction levels, operational actions) of each $p \in F$ is $B_{p}$. For all $\lambda \in B^{F}, \lambda$ could be treated as a multi-choice coalition. A multi-choice coalition 
$\lambda$ could also be treated as a combination of reaction levels for catalysts that are meant to evaluate some goals, which are coincident to its factors. The usability of a multi-choice coalition $\lambda$ (i.e., $C(\lambda)$ ) with the resource it needs for its activities is done by the factors and the grade of relationship of factor $p \in F$ to multi-choice coalition $\lambda$ is computed by the collection $B_{p}$ of the active levels (reaction levels, operational actions) of factor $p$. Observe that this notion of computing the grade of relationship is different from the more general one, in which the grade of relationship is computed by the share of the coalitional resource that a factor owns. It better reflects that the pressure factors are ready to take over when putting in reaction processes of combinations for catalysts and also its individual ability in realizing the goals that the reaction processes of combinations for catalysts are meant to reach. In this article, we adopt the characterizations of the MLII to demonstrate that the MLII could be applied to interpret the relationship grade of a factor to a multi-choice coalition as a measure of the pressure that the factor assumes by transferring a part of its resource to the coalition considered as reaction processes of combinations for catalysts.

Example 2. Glyphosate and glufosinate-ammonium (Gla) are non-specific herbicides that are widely used to protect crops from the adverse effect of weed species competition. The molecular mechanism of the actions of these herbicides is to block the enzyme-catalyzed action. There are standardized allowable amounts and toxicological evaluation procedures under simple environmental conditions. However, in modern times, the phenomenon of compound environmental pollution that is caused by multiple pollution sources is quite common. Evidence reveals that the toxicity of herbicide compounds to aquatic organisms varies due to the catalytic effects of plastic particles in the environment, bringing a great deal of complexity to compound toxicity in aquatic environments. Thus, one could suppose that $F$ is the set of herbicides and plastic particles. Further, let the amount of the reaction levels of each $p \in F$ is $B_{p}$. A multi-choice coalition $\lambda \in B^{F}$ could also be treated as a combination of reaction levels for herbicides and plastic particles that are meant to evaluate some effects, which are coincident to its factors. The effect of a multi-choice coalition $\lambda(i . e ., C(\lambda)$ ) with the reaction that it causes for its activities is done by the factors and the level of relationship of factor $p \in F$ to multi-choice coalition $\lambda$ is determined by the collection $B_{p}$ of the reaction levels of factor $p$. Observe that this notion of determining the level of relationship is different from the more general one, in which the level of relationship is determined by the share of coalitional reaction a factor owns. Based on the notion of multi-choice TU circumstances, the aquatic environment containing the plastic particle complexity could be constructed to be $(F, C)$. By applying the characterizations of the MLII, the toxicological effects and related synergistic effects are collected and evaluated under the mode that plastic particles and multiple herbicides exert multiple actions. It is expected that an assessment model for the compound polluted environment will eventually be developed by combining experimental data with the game-theoretical results of the MLII.

Example 3. On the other hand, toxic heavy metal pollutants have become very common environmental pollutants, which are highly toxic to the aquatic environment due to their persistence in the environment. Therefore, in addition to the toxicity of pesticides, heavy metals are also the main pollutants in the aquatic environment today. Numerous studies have been conducted in order to investigate the impact of high concentrations of multiple heavy metal mixtures on physiological, biochemical, and behavioral changes of aquatic organisms by using the aquatic organism system that is sensitive to environmental toxicity as the assessment platform. Such studies explore the possible toxic effects and environmental and ecological risks to aquatic organisms when they enter the environmental water ecosystem. Suppose that $F$ is the set of heavy metal pollutants and mitigating catalysts. Further, let the amount of the reaction grades of each $p \in F$ is $B_{p}$. A multi-choice coalition $\lambda \in B^{F}$ could also be treated as a combination of reaction grades for heavy metal pollutants and mitigating catalysts that are meant to evaluate some impacts or effects, which are coincident to its factors. The synergistic effects of a multi-choice coalition $\lambda$ of multiple toxicants and multiple mitigating catalysts after the application of metal chelators and antioxidants (i.e., $C(\lambda)$ ) are evaluated under the mode that multiple toxicants and mitigating catalysts both exert multiple types of actions. Based on the framework of multi-choice TU circumstance, a toxicity-reacting environment with high concentrations of multiple heavy metal mixtures and mitigating catalysts could be constructed to be $(F, C)$. It is expected that the game-theoretical results of the MLII could 
be used to generate balanced configurations for analyzing the toxicity of heavy metal mixtures and the toxicity mitigation procedures of metal chelators and antioxidants.

\section{Game-Theoretical Characterizations}

In order to present the rationality of the MLII, we demonstrate that there is a reduction that could be applied to characterize the MLII in this section. Some more properties are needed. Let $\tau$ be a power index on $\Psi$.

- $\tau$ contents level completeness (LCOM) if $\sum_{p \in F} \sum_{q=1}^{b_{p}} \tau_{p, q}(F, C)=C(b)$ for all $(F, C) \in \Psi$.

- $\quad \tau$ contents criterion for circumstances property (CFCP) if $\tau(F, C)=\bar{\gamma}(F, C)$ for all $(F, C) \in \Psi$ with $|F| \leq 2$.

- $\quad \tau$ contents level equal effect property (LEEP) if for all $(F, C) \in \Psi$ with $C\left(\lambda, k_{p}, 0\right)$ $C\left(\lambda, k_{p}-1,0\right)=C\left(\lambda, 0, k_{h}\right)-C\left(\lambda, 0, k_{h}-1\right)$ for some $\left(p, k_{p}\right),\left(h, k_{h}\right) \in L^{F}$ and for all $\lambda \in B^{F \backslash\{p, h\}}, \tau_{p, k_{p}}(F, C)=\tau_{h, k_{h}}(F, C)$.

- $\tau$ contents level synchronization (LSYN) if for all $(F, C),(F, D) \in \Psi$ with $C(\lambda)=$ $D(\lambda)+\sum_{p \in A(\lambda)} \sum_{q=1}^{\lambda_{p}} \mu_{p, q}$ for some $\mu \in \mathbb{R}^{L^{F}}$ and for all $\lambda \in B^{F}, \tau(F, C)=\tau(F, D)+\mu$.

LCOM states that all of the factors distribute all the usability entirely when all factors react at all levels in a circumstance. CFCP is a self-sufficient condition when there is only one factor in the circumstance, but when there are two factors in the circumstance, each of them first takes back what they could have produced alone and, at the end of the circumstance, they share all of the remaining profits and losses. LEEP states that the effects of two factors should be equal if the marginal distinctions of these two factors are coincident. LSYN can be asserted as an extreme weakness of additivity. In Section 4, the interaction between above game-theoretical properties and reaction processes of combinations for catalysts will be provided in detail. By Definition 1, it is easy to clarify the MLII contents LCOM, CFCP, LEEP, and LSYN.

A natural generalized form of the reduction due to Hsieh and Liao [11] is as follows. Given $(F, C) \in \Psi, S \subseteq F$ and a power index $\tau$, the reduced circumstance $\left(S, C_{S}^{\tau}\right)$ related to $S$ and $\tau$ is defined by for all $\lambda \in B^{S}$,

$$
= \begin{cases}C_{S}^{\tau}(\lambda) & \lambda=0_{S}, \\ C\left(\lambda, 0_{F \backslash\{p\}}\right) & S \geq|2|, A(\lambda)=\{p\} \text { for some } p, \\ C\left(\lambda, b_{F \backslash S}\right)-\sum_{p \in F \backslash S} \sum_{q=1}^{b_{p}} \tau_{p, q}(F, C) & \text { otherwise. }\end{cases}
$$

The consonance requirement may be stated informally, as follows. Let $\tau$ be a power index on $\Psi$. For any couple of two factors in a circumstance, one introduces a "reduced circumstance" among them by considering the amounts remaining after the rest of the factors are given the effects prescribed by $\tau$. Subsequently $\tau$ is called consonant if it always yields the same effects as in the initial circumstance when it is applied to an arbitrary reduced circumstance. Formally, a power index $\tau$ contents consonance (CSE) if for all $(F, C) \in \Psi$ with $|F| \geq 3$, for all $S \subseteq F$ with $|S|=2$ and for all $\left(p, k_{p}\right) \in L^{S}$, $\tau_{p, k_{p}}(F, C)=\tau_{p, k_{p}}\left(S, C_{S}^{\tau}\right)$.

In the following, some results that are related to the CSE property are introduced.

Lemma 1. The MLII $\bar{\gamma}$ contents CSE. 
Proof. Let $(F, C) \in \Psi$ with $|F| \geq 3$ and $S \subseteq F$ with $|S|=2$. Assume that $S=\{p, h\}$. By the definition of $\bar{\gamma}$, for all $\left(p, k_{p}\right) \in L^{S}$,

$$
\bar{\gamma}_{p, k_{p}}\left(S, C_{S}^{\bar{\gamma}}\right)=\gamma_{p, k_{p}}\left(S, C_{S}^{\bar{\gamma}}\right)+\frac{1}{\left\|b_{S}\right\|} \cdot\left[C_{S}^{\bar{\gamma}}\left(b_{S}\right)-\sum_{t \in S} \sum_{q=1}^{b_{t}} \gamma_{t, q}\left(S, C_{S}^{\bar{\gamma}}\right)\right] .
$$

By definitions of $\gamma$ and $C_{S}^{\bar{\gamma}}$, for all $k_{p} \in B_{p}^{+}$,

$$
\begin{aligned}
\gamma_{p, k_{p}}\left(S, C_{S}^{\bar{\gamma}}\right) & =C_{S}^{\bar{\gamma}}\left(k_{p}, 0\right)-C_{S}^{\bar{\gamma}}\left(k_{p}-1,0\right) \\
& =C\left(k_{p}, 0_{F \backslash\{p\}}\right)-C\left(k_{p}-1,0_{F \backslash\{p\}}\right) \\
& =\gamma_{p, k_{p}}(F, C) .
\end{aligned}
$$

Hence, by Equations (1) and (2), and definitions of $C_{S}^{\bar{\gamma}}$ and $\bar{\gamma}$,

$$
\begin{aligned}
& \bar{\gamma}_{p, k_{p}}\left(S, C_{S}^{\bar{\gamma}}\right) \\
= & \gamma_{p, k_{p}}(F, C)+\frac{1}{\|S\|} \cdot\left[C_{S}^{\bar{\gamma}}\left(b_{S}\right)-\sum_{t \in S} \sum_{q=1}^{b_{t}} \gamma_{t, q}(F, C)\right] \\
= & \gamma_{p, k_{p}}(F, C)+\frac{1}{\|S\|} \cdot\left[C(b)-\sum_{t \in F \backslash S} \sum_{q=1}^{b_{t}} \bar{\gamma}_{t, q}(F, C)-\sum_{t \in S} \sum_{q=1}^{b_{t}} \gamma_{t, q}(F, C)\right] \\
= & \gamma_{p, k_{p}}(F, C)+\frac{1}{\|S\|} \cdot\left[\sum_{t \in S} \sum_{q=1}^{b_{t}} \bar{\gamma}_{t, q}(F, C)-\sum_{t \in S} \sum_{q=1}^{b_{t}} \gamma_{t, q}(F, C)\right] \\
= & \gamma_{p, k_{p}}(F, C)+\frac{1}{\|S\|} \cdot[\|S\| \\
= & \left.\gamma_{p, k_{p}}(F, C)+\frac{1}{\|N\|} \cdot\left[C(b)-\sum_{t \in F} \sum_{q=1}^{b_{t}} \gamma_{t, q}(F, C)\right]\right] \\
= & \bar{\gamma}_{p, k_{p}}(F, C) .
\end{aligned}
$$

Similarly, $\bar{\gamma}_{h, k_{h}}\left(S, C_{S}^{\bar{\gamma}}\right)=\bar{\gamma}_{h, k_{h}}(F, C)$ for all $k_{h} \in B_{h}^{+}$. Accordingly, the MLII contents CSE.

Lemma 2. If a power index $\tau$ contents CFCP and CSE, then it also contents LCOM.

Proof. Let $\tau$ be a power index on $\Psi$ contenting CFCP and CSE, and $(F, C) \in \Psi$. It is trivial for $|F| \leq 2$ by CFCP. Assume that $|F| \geq 3$. Let $h \in F$ consider the reduced circumstance $\left(\{h\}, C_{\{h\}}^{\tau}\right)$. By definition of $C_{\{h\}}^{\tau}$,

$$
C_{\{h\}}^{\tau}\left(b_{h}\right)=C(b)-\sum_{p \in F \backslash\{h\}} \sum_{q=1}^{b_{p}} \tau_{p, q}(F, C) .
$$

Because $\tau$ contents CSE, $\tau_{h, k_{h}}(F, C)=\tau_{h, k_{h}}\left(\{h\}, C_{\{h\}}^{\tau}\right)$ for all $k_{h} \in B_{h}$. In particular, $\tau_{h, b_{h}}(F, C)=\tau_{h, b_{h}}\left(\{h\}, C_{\{h\}}^{\tau}\right)$. On the other hand, by CFCP of $\tau, \sum_{q=1}^{b_{h}} \tau_{h, j}(F, C)=C_{\{h\}}^{\tau}\left(b_{h}\right)$.

Hence, $\sum_{p \in F} \sum_{q=1}^{b_{p}} \tau_{p, q}(F, C)=C(b)$, i.e., $\tau$ contents LCOM.

Subsequently, we characterize the MLII by criterion for circumstances property and consonance.

Theorem 1. A power index $\tau$ on $\Psi$ contents CFCP and CSE if and only if $\tau=\bar{\gamma}$.

Proof. By Lemma 1, $\bar{\gamma}$ contents CSE. Clearly, $\bar{\gamma}$ contents CFCP. 
In order to demonstrate uniqueness, suppose that $\tau$ contents CFCP and CSE on $\Psi$. By Lemma 2, $\tau$ also contents LCOM. Let $(F, C) \in \Psi$. If $|F| \leq 2$, then by CFCP of $\tau$, $\tau(F, C)=\bar{\gamma}(F, C)$. The case $|F|>2$ : let $p \in F$ and $S=\{p, q\}$ for some $q \in F \backslash\{p\}$, then for all $k_{p} \in B_{p}^{+}, k_{q} \in B_{q}^{+}$,

$$
\begin{aligned}
& \tau_{p, k_{p}}(F, C)-\tau_{j, k_{q}}(F, C) \\
= & \tau_{p, k_{p}}\left(S, C_{S}^{\tau}\right)-\tau_{j, k_{q}}\left(S, C_{S}^{\tau}\right) \\
= & \bar{\gamma}_{p, k_{p}}\left(S, C_{S}^{\tau}\right)-\bar{\gamma}_{j, k_{q}}\left(S, C_{S}^{\tau}\right) \\
= & \gamma_{p, k_{p}}\left(S, C_{S}^{\tau}\right)-\gamma_{j, k_{q}}\left(S, C_{S}^{\tau}\right) \\
= & {\left[C_{S}^{\tau}\left(k_{p}, 0\right)-C_{S}^{\tau}\left(k_{p}-1,0\right)-C_{S}^{\tau}\left(0, k_{q}\right)+C_{S}^{\tau}\left(0, k_{q}-1\right)\right] } \\
= & {\left[C\left(k_{p}, 0_{F \backslash\{p\}}\right)-C\left(k_{p}-1,0_{F \backslash\{p\}}\right)-C\left(0_{F \backslash\{q\}}, k_{q}\right)+C\left(0_{F \backslash\{q\}}, k_{q}-1\right)\right] . }
\end{aligned}
$$

Similarly, $\bar{\gamma}$ instead of $\tau$ in Equation (3), we have that

$$
\begin{aligned}
& \bar{\gamma}_{p, k_{p}}(F, C)-\bar{\gamma}_{j, k_{q}}(F, C) \\
= & {\left[C\left(k_{p}, 0_{F \backslash\{p\}}\right)-C\left(k_{p}-1,0_{F \backslash\{p\}}\right)-C\left(0_{F \backslash\{q\}}, k_{q}\right)+C\left(0_{F \backslash\{q\}}, k_{q}-1\right)\right] . }
\end{aligned}
$$

By Equations (3) and (4),

$$
\tau_{p, k_{p}}(F, C)-\tau_{j, k_{q}}(F, C)=\bar{\gamma}_{p, k_{p}}(F, C)-\bar{\gamma}_{j, k_{q}}(F, C)
$$

This implies that $\tau_{p, k_{p}}(F, C)-\bar{\gamma}_{p, k_{p}}(F, C)=a$ for all $\left(p, k_{p}\right)$. It remains to demonstrated that $a=0$. By LCOM of $\tau$ and $\bar{\gamma}$,

$$
0=\sum_{p \in F} \sum_{k_{p}=1}^{b_{p}}\left[\tau_{p, k_{p}}(F, C)-\bar{\gamma}_{p, k_{p}}(F, C)\right]=\|b\| \cdot a .
$$

That is, $a=0$.

Next, we characterize the MLII by means of level completeness, level equal effect property, level synchronization, and consonance.

Lemma 3. If a power index $\tau$ on $\Psi$ contents LCOM, LEEP, and LSYN, then $\tau$ contents CFCP.

Proof. Assume that a power index $\tau$ contents LCOM, LEEP, and LSYN. Given $(F, C) \in \Psi$ with $F=\{p, h\}$ for some $p \neq h$. We define a circumstance $(F, D)$ to be that for all $\lambda \in B^{F}$,

$$
D(\lambda)=C(\lambda)-\sum_{t \in A(\lambda)} \sum_{q=1}^{\lambda_{t}} \gamma_{t, q}(F, C) .
$$

By definition of $D, D\left(k_{p}, 0\right)-D\left(k_{p}-1,0\right)=0$ for all $k_{p} \in B_{p}^{+}$. Similarly, $D\left(k_{h}, 0\right)-D\left(k_{h}-\right.$ $1,0)=0$ for all $k_{h} \in B_{h}^{+}$. Because $D\left(k_{p}, 0\right)-D\left(k_{p}-1,0\right)=0=D\left(k_{h}, 0\right)-D\left(k_{h}-1,0\right)$, by LEEP of $\tau, \tau_{p, k_{p}}(F, D)=\tau_{h, k_{h}}(F, D)$. By LCOM of $\tau$,

$$
D(b)=\sum_{q=1}^{b_{p}} \tau_{p, q}(F, D)+\sum_{q=1}^{b_{h}} \tau_{h, q}(F, D)=\|b\| \cdot \tau_{p, q}(F, D)
$$

for all $q \in B_{p}^{+}$. Therefore, by Equation (5) and definition of $D$,

$$
\tau_{p, q}(F, D)=\frac{D(b)}{\|b\|}=\frac{1}{\|b\|} \cdot\left[C(b)-\sum_{t \in F} \sum_{q=1}^{b_{t}} \gamma_{p, q}(F, C)\right]
$$


By LSYN of $\tau$,

$$
\begin{aligned}
\tau_{p, k_{p}}(F, C) & =\tau_{p, k_{p}}(F, D)+\gamma_{p, k_{p}}(F, C) \\
& =\frac{1}{\|b\|} \cdot\left[C(b)-\sum_{t \in F} \sum_{q=1}^{b_{t}} \gamma_{t, q}(F, C)\right]+\gamma_{p, k_{p}}(F, C) \\
& =\bar{\gamma}_{p, k_{p}}(F, C) .
\end{aligned}
$$

Similarly, $\tau_{h, k_{h}}(F, C)=\bar{\gamma}_{h, k_{h}}(F, C)$ for all $k_{h} \in B_{h}^{+}$. Hence, $\tau$ contents CFCP.

Theorem 2. A power index $\tau$ on $\Psi$ contents LCOM, LEEP, LSYN, and CSE, if and only if $\tau=\bar{\gamma}$.

Proof. By Definition $1, \bar{\gamma}$ contents LCOM, LEEP, and LSYN. The remaining proofs could be finished by Theorem 1 and Lemmas 1,3 .

\section{Discussion on Reaction Processes of Combinations for Catalysts}

Based on the rapidly changing interactions, this article adopts game-theoretical results to analyze the accuracy, plausibility, and feasibility of different combinations for catalysts by inquiring "how is the combination generated", "why do we choose this combination", "is such combination accurate", and "how efficient is such combination".

In previous sections, it is demonstrated that the main advantage of the MLII and related characterization is that the MLII of a multi-choice TU circumstance absolutely exists and it generates an exact effect for a given factor reacting at a given active level that is different from the usual viewpoint with multi-choice TU circumstances, which determines a type of global effect for a given factor by collecting the distinctions of this factor among its all active levels.

One would like to expect that the MLII could offer "optimal outcome" or "balanced outcome" exactly under reaction processes of combinations for catalysts. We further explore the interaction between game-theoretical properties and reaction processes of combinations for catalysts in order to illustrate how the MLII could be applied and to increase its meaning more transparently.

1. Level completeness: balanced catalyst-relating processes must make full use of environmental resources. Therefore, a balanced catalyst-relating process should satisfy the completeness property.

2. Criterion for circumstances property: catalysts have their own properties of actions. Interactions between catalysts are generally derived from two-catalyst interactions, followed by group interactions. Accordingly, a balanced catalyst-relating process should satisfy the criterion for circumstances property.

3. Level equal effect property: if any two catalysts are equally beneficial to the overall environment after the action of catalyst grouping, the influence of these two catalysts on the overall environment should also be equal. Therefore, a balanced catalystrelating process should satisfy the level equal effect property.

4. Level synchronization: balanced catalyst-relating processes, in which each catalyst is applied with the right proportion to achieve the target action, rather than the amount (large or small), depending on the target action, which should achieve the most balanced effect in accordance with the proportionality principle. Hence, a balanced catalyst-relating process should satisfy the level synchronization property.

5. Consonance: balanced catalyst-relating processes are verified through a continuous iterative synergistic procedure, and it should bring consistent benefits. Therefore, a balanced catalyst-relating process should satisfy the consonance property.

By applying related definitions, statements, and examples of Section 2, one could see that the framework of reaction processes of combinations for catalysts could be formulated as a multi-choice TU circumstance. Based on Theorems 1 and 2 in Section 3, it is demonstrated that the MLII is the only mechanism contenting level completeness, criterion for circumstances property, level equal effect property, level synchronization, and conso- 
nance simultaneously. Based on the statements $1-5$, it is clear to see that the properties of level completeness, criterion for circumstances property, level equal effect property, level synchronization, and consonance should be essential conditions in the context of reaction processes of combinations for catalysts. Therefore, the MLII might be applied to be an useful distribution notion in the context of reaction processes of combinations for catalysts.

In the following, we present an application with real data. Let $(F, C) \in \Psi$ be a aquatic environment with catalysts set $F=\{p, q, k\}$ and active level vector $b=(2,1,1)$. Further, let $C(2,1,1)=5, C(1,1,1)=4, C(2,1,0)=6, C(2,0,1)=5, C(2,0,0)=9$, $C(1,1,0)=2, C(1,0,1)=-7, C(0,1,1)=3, C(1,0,0)=-1, C(0,1,0)=2, C(0,0,1)=-3$ and $C(0,0,0)=0$ be the usability that the factors can occur under all reaction situations. By Definition 1,

$$
\begin{array}{rrrrr}
\gamma_{p, 2}(F, C) & =10, \quad \gamma_{p, 1}(F, C) & = & -1, \\
\gamma_{q, 1}(F, C) & =2, \quad \gamma_{k, 1}(F, C) & = & -3, \\
\overline{\gamma_{p, 2}}(F, C) & =9.25, \quad \overline{\gamma_{p, 1}}(F, C) & = & -1.75, \\
\overline{\gamma_{q, 1}}(F, C) & =1.25, \quad \frac{\gamma_{k, 1}}{\gamma}(F, C) & = & -3.75 .
\end{array}
$$

It is clear to see the effect or the power of each factor when it reacts with a specific level in $(F, C)$. For example, the effect of factor $p$ is $\overline{\gamma_{p, 2}}(F, C)=9.25$ when $p$ reacts with the level 2 in $(F, C)$.

\section{Concluding Remarks}

1. The purpose of this article is to propose some generalizations and applications to the existing combinatorial approaches for catalyst-relating processes.

- A generalization of the PEANSC, the multi-choice level-individual index, is proposed by simultaneously focusing on the factors and its active levels.

- We provide two characterizations in order to analyze the accuracy and the rationality of the multi-choice level-individual index.

- In conjunction with the constructed game-theoretical results that are related to chemical and biochemical environments, we further analyze, verify, and demonstrate the validity, accuracy, feasibility, plausibility, and applicability of the combination of catalysts with different conditions and actions by applying some examples and related interpretations.

2. By both considering the factors and its active levels (actions), Hwang and Liao [12] defined an extended Shapley value [13] and related axiomatic results on fuzzy TU circumstances. One should also compare our results with the results due to Hwang and Liao [12]. There are several major dissimilarities:

- The MLII and related results are initially introduced.

- Power indexes on traditional TU circumstances have only discussed participation or non-participation of all factors. In this paper, we propose the power index for analyzing the distribution mechanism under multi-choice behavior.

- Under multi-choice TU circumstances, pre-existing power indexes have been adopted in order to determine a type of global effect for a given factor by collecting the distinctions of this factor among its all active levels. By considering real-world situations, we propose the MLII to evaluate the related effects by both considering a given factor reacting at a given active level.

- The game-theoretical results of this article are adopted to evaluate the reaction processes of combinations for catalysts. This application does not appear in pre-existing results.

3. The results that have been proposed in this article raise one motivation.

- whether some more game-theoretical results could be adopted to analyze, construct, simulate, and derive the most efficient optimal or balanced combinations for a group of catalysts with different conditions and actions.

This is left to the readers. 
Author Contributions: Conceptualization, K.H.-C.C., J.-C.H. and Y.-H.L.; methodology, K.H.-C.C., J.-C.H. and Y.-H.L.; validation, K.H.-C.C., J.-C.H. and Y.-H.L.; formal analysis, K.H.-C.C., J.-C.H. and Y.-H.L.; investigation, K.H.-C.C., J.-C.H. and Y.-H.L.; writing-original draft preparation, Y.-H.L.; writing-review and editing, K.H.-C.C., J.-C.H. and Y.-H.L.; project administration, K.H.-C.C., J.-C.H. and Y.-H.L. All authors have read and agreed to the published version of the manuscript.

Funding: This study was funded by National Pingtung University NPTU-110-003.

Conflicts of Interest: The authors declare no conflict of interest.

\section{References}

1. Everbroeck, T.V.; Ciocarlan, R.-G.; Hoey, W.V.; Mertens, M.; Cool, P. Copper-containing mixed metal oxides (Al, Fe, Mn) for application in three-way catalysis. Catalysts 2020, 10, 1344. [CrossRef]

2. Gebresillase, M.N.; Raguindin, R.Q.; Kim, H.; Seo, J.G. Supported bimetallic catalysts for the solvent-free hydrogenation of levulinic acid to $\gamma$-valerolactone: Effect of metal combination ( $\mathrm{Ni}-\mathrm{Cu}, \mathrm{Ni}-\mathrm{Co}, \mathrm{Cu}-\mathrm{Co}$ ). Catalysts 2020, 10, 1354. [CrossRef]

3. Losada-Garcia, N.; Cabrera, Z.; Urrutia, P.; Garcia-Sanz, C.; Andreu, A.; Palomo, J.M. Recent advances in enzymatic and chemoenzymatic cascade processes. Catalysts 2020, 10, 1258. [CrossRef]

4. Malgas, S.; Pletschke, B.I. Combination of CTec2 and GH5 or GH26 endo-mannanases for effective lignocellulosic biomass degradation. Catalysts 2020, 10, 1193. [CrossRef]

5. Ntana, F.; Mortensen, U.H.; Sarazin, C.; Figge, R. Aspergillus: A powerful protein production platform. Catalysts 2020, 10, 1064. [CrossRef]

6. Pérez-Mayoral, E.; Matos, I.; Bernardo, M.; Ventura, M.; Fonseca, I.M. Carbon-based materials for the development of highly dispersed metal catalysts: Towards highly performant catalysts for fine chemical synthesis. Catalysts 2020, 10, 1407. [CrossRef]

7. Hwang, Y.A.; Liao, Y.H. The unit-level-core for multi-choice games: The replicated core for TU games. J. Glob. Optim. 2010, 47, 161-171. [CrossRef]

8. Liao, Y.H. The maximal equal allocation of nonseparable costs on multi-choice games. Econ. Bull. 2008, 3, 1-8.

9. Liao, Y.H. The duplicate extension for the equal allocation of nonseparable costs. Oper. Res. Int. J. 2012, 13, 385-397. [CrossRef]

10. van den Nouweland, A.; Potters, J.; Tijs, S.; Zarzuelo, J. Core and related solution concepts for multi-choice games. ZOR-Math. Methods Oper. Res. 1995, 41, 289-311. [CrossRef]

11. Hsieh, Y.L.; Liao, Y.H. The Pseudo EANSC: Axiomatization and Dynamic Process. Master's Thesis, National Pingtung University, Pingtung City, Taiwan, 2016.

12. Hwang, Y.A.; Liao, Y.H. The consistent value of fuzzy games. Fuzzy Sets Syst. 2009, 160, 644-656. [CrossRef]

13. Shapley, L.S. A value for n-person game. In Distinctions to the Theory of Games II; Kuhn, H.W., Tucker, A.W., Eds.; Princeton University Press: Princeton, NJ, USA, 1953; pp. 307-317. 